\title{
Case Files of the Medical Toxicology Fellowship of the California Poison Control System-San Francisco: Calcium Plus Digoxin-More Taboo than Toxic?
}

\author{
Christian P. Ericksona, MD, MPH, Kent R. Olson, $M D^{b, c}$
}

aPostgraduate Fellow in Medical Toxicology, University of California, San Francisco

bMedical Director, San Francisco Division, California Poison Control System

cClinical Professor of Medicine and Pharmacy, University of California, San Francisco

\section{CASE PRESENTATION}

A 68-year-old woman with a history of diabetes mellitus presented to the emergency department after experiencing several days of nausea and vomiting. Recorded vital signs included: blood pressure $98 / 32 \mathrm{~mm}$ $\mathrm{Hg}$, heart rate 69 bpm, "normal" respirations, and no fever. Her initial ECG revealed peaked $T$ waves and regular rhythm with no visible $P$ waves. Initial serum electrolytes were notable for a potassium level of $7.7 \mathrm{mmol} / \mathrm{L}$, and a creatinine level of $9.6 \mathrm{mg} / \mathrm{dL}(849 \mu \mathrm{mol} / \mathrm{L})$. She was unable to provide a list of her current medications or other medical history. There was no old chart immediately available for comparison.

\section{What are the effects of elevated potassium on the heart?}

As in other tissues, most sodium in the myocardium is extracellular, and most potassium is intracellular. The concentrations of each are maintained by the $\mathrm{Na}^{+}-\mathrm{K}^{+}$-ATPase pump and are also dependent on the relative permeability of the cell surface to migration of $\mathrm{Na}^{+}$or $\mathrm{K}^{+}$down their respective concentration gradients. In a normal cardiac cell, the resting potential during diastole is approximately $-90 \mathrm{mV}$. If $\mathrm{Na}^{+}$enters the cell, the electrical potential becomes less negative; conversely, if $\mathrm{K}^{+}$leaves the cell the transmembrane potential becomes more negative $[1,2]$.

Electrical signals are propagated through the heart by action potentials that open voltage-gated sodium channels, allowing rapid influx of $\mathrm{Na}^{+}$and depolarization of the cell (phase 0), as

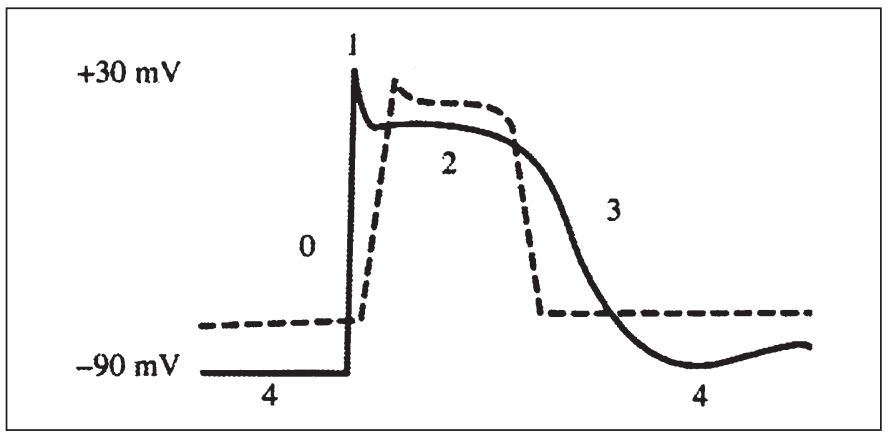

Figure 1: Cardiac action potential

depicted in Figure 1. The rate of rise of phase 0 is directly proportional to the number of sodium channels that are opened during depolarization. The more negative the resting membrane potential at the start of phase 0 , the greater the number of $\mathrm{Na}^{+}$channels in the "ready-to-open" state. Conversely, if the resting membrane potential is less negative than normal, fewer $\mathrm{Na}^{+}$ channels are ready to open and depolarization will be less robust, with reduced upstroke velocity $\left(\mathrm{V}_{\text {max }}\right)$, reduced action potential amplitude, and reduced conduction velocity.

As the membrane potential reaches -40 to $-45 \mathrm{mV}$ during phase 0 depolarization, voltage-gated calcium channels are opened, allowing calcium entry. At about the same time, potassium channels open and allow potassium to exit the cell, balancing the influx of calcium. Together, the opposing effects of calcium influx

Keywords: calcium, digoxin, cardiac glycosides, potassium, dysrhythmias

Notes: There was no outside funding of any kind used for this study.

Acknowledgments: The authors wish to thank Dr. Bertram G. Katzung for his review of the manuscript and invaluable assistance with our understanding ion channels in cardiac cells.

Corresponding Author: Kent R. Olson, MD, UCSF Box 1369, San Francisco, CA 94143-1369. (e-mail: olson@calpoison.org) 
and potassium efflux account for the relatively flat slope of phase 2 of the action potential. When the calcium channels close, continued potassium efflux leads to repolarization (phase 3 ) of the cell back to its resting potential at about $-90 \mathrm{mV}$.

For reasons that are not fully understood, the potassium channels (known as inward potassium rectifier channels, or $I_{\mathrm{Kr}}$ ) are sensitive to external concentrations of $\mathrm{K}^{+}$and exhibit increased permeability to $\mathrm{K}^{+}$in the presence of hyperkalemia [1]. Given that the concentration gradient favors potassium efflux, hyperkalemia causes $\mathrm{K}^{+}$to leave the cell more rapidly, and phase 3 is more sharply sloped and the overall length of the action potential is shortened.

Rising levels of potassium outside the cell lower the transmembrane potential for potassium, which in turn reduces the resting membrane potential (makes it less negative). This can bring the cell close enough to threshold to cause a spontaneous depolarization, increasing excitability. However, as the transmembrane potential becomes progressively less negative there are fewer $\mathrm{Na}^{+}$ channels in a state of readiness to open during phase 0 . This slows the rate of $\mathrm{Na}^{+}$entry and reduces $\mathrm{V}_{\text {max }}$ and conduction velocity. Thus, with severe hyperkalemia, conduction may be seriously impaired or blocked completely [1,2]; the QRS complex becomes broad and looks like a "sine-wave," then flattens into asystole.

The effects of hyperkalemia on potassium permeability are particularly important in pacemaker cells (e.g., the sinoatrial [SA] and atrioventricular $[\mathrm{AV}]$ nodes and ectopic pacemakers). In these cells, gradual depolarization of the cell normally occurs as a result of slow entry of sodium and calcium ions during phase 4 . When threshold is reached, spontaneous depolarization occurs, largely due to rapid $\mathrm{Ca}^{++}$entry. This predictable event provides the automatic "pacemaker" action potential, and the slope of phase 4 determines the sinus rate. At normal potassium concentrations, the $I_{\mathrm{Kr}}$ channels in pacemaker cells are not highly permeable to potassium; therefore, the influx of $\mathrm{Na}^{+}$and $\mathrm{Ca}^{++}$is not fully balanced by efflux of $\mathrm{K}^{+}$, allowing gradual depolarization to occur. On the other hand, the increase in $\mathrm{K}^{+}$permeability associated with hyperkalemia can have a dramatic effect, counteracting the influx of positive charges and slowing or stopping pacemaker cell depolarization [1].

In summary, severe hyperkalemia slows impulse transmission in conducting tissues, resulting in progressive widening of the PR and QRS intervals, and disturbs impulse formation in pacemaker cells, leading to loss of $\mathrm{P}$ waves and, ultimately, asystole. In experimental models, the earliest manifestation of hyperkalemia is narrow (150-250 msec) peaked $\mathrm{T}$ waves, which usually appear at serum potassium levels greater than $5.5 \mathrm{mmol} / \mathrm{L}$. Shortening of the PR and QT intervals may also occur, along with increased myocyte excitability [2]. At levels above $6.5 \mathrm{mmol} / \mathrm{L}$, phase 0 is slowed, the QRS complex widens, and the PR interval is prolonged. At higher levels $(8-10 \mathrm{mmol} / \mathrm{L}), \mathrm{SA}$ impulse formation is absent (loss of $P$ waves) and an idioventricular rhythm may occur. The QRS interval continues to widen and eventually blends with the T wave, producing a "sine-wave" pattern, presaging ventricular fibrillation or asystole [2].
Clinically, there is relatively poor correlation of the ECG with serum potassium levels. In one study, only $55 \%$ of patients with serum potassium greater than $6.8 \mathrm{mmol} / \mathrm{L}$ had ECG changes consistent with hyperkalemia [3]. In another study, physicians were able to predict hyperkalemia from the ECG with a sensitivity of only $35-43 \%$ and a specificity of $85-86 \%$ [4].

\section{What are the causes of hyperkalemia?}

Renal failure and administered medications are the most common causes [3]. Drugs and poisons associated with hyperkalemia include ACE inhibitors, spironolactone, digitalis glycosides, fluoride, potassium supplements, succinylcholine, and beta-adrenergic blockers [5-8]. Other causes include severe rhabdomyolysis, massive hemolysis, and tumor lysis syndrome. Factitious hyperkalemia is common due to hemolysis during blood draw under pressure.

\section{What are the available treatments for hyperkalemia?}

Besides discontinuing offending medications and treating underlying medical conditions, there are three basic strategies for acute management of hyperkalemia: move $\mathrm{K}^{+}$from the extracellular to the intracellular space (e.g., glucose plus insulin, $\beta_{2}$-agonists, sodium bicarbonate); enhance elimination of total body $\mathrm{K}^{+}$(e.g., dialysis, sodium polystyrene sulfonate [e.g., Kayexalate]); and antagonize the physiologic effects of $\mathrm{K}^{+}$(e.g., calcium). We'll focus on calcium, which has the most direct and rapid effect on the electrophysiologic manifestations of hyperkalemia and which is at the center of the treatment controversy for hyperkalemia in patients with possible digitalis poisoning.

Calcium is thought to antagonize the effects of hyperkalemia in the following ways:

1. Calcium increases the threshold for depolarization and can reduce the excitability that occurs with moderate hyperkalemia [2]. This mechanism is thought to involve calcium binding to negatively charged sites on the outer surface of the membrane, especially the mouths of the sodium channels themselves, thereby increasing the "effective" resting transmembrane potential that controls the gating of these channels and making them difficult to activate [9]. For example, if the transmembrane potential is $-90 \mathrm{mV}$ but there is also $-10 \mathrm{mV}$ worth of negatively charged groups on the outer cell membrane, there is actually an "effective" transmembrane potential of only $-80 \mathrm{mV}(-90$ minus $-10=-80)$. If the negatively charged groups on the outer membrane are neutralized by administering a divalent cation such as calcium (or magnesium), the effective transmembrane potential is now increased to $-90 \mathrm{mV}(-90$ minus $0=-90)$. This reduces hyperexcitability and also increases the number of available sodium channels, improving conduction. Animal studies show that increasing calcium levels increases $\mathrm{V}_{\max }$ (the rate of rise of phase 0 of the action potential), improving conduction in Purkinje fibers [10]. 
2. In pacemaker cells and the AV node, an increase in extracellular calcium increases the concentration gradient across the membrane and increases calcium influx, promoting pacemaker function and impulse propagation [11].

Calcium's electrophysiologic effects are rapid and its use is indicated when evidence of membrane depression is present (e.g., loss of $\mathrm{P}$ waves, wide QRS, sine-wave pattern). Note that calcium does not lower the serum potassium level and its effects are of short duration (30-60 minutes) $[2,6,8]$, thus steps should also be taken to enhance potassium movement into cells and to eliminate excess total body potassium.

Transfer of potassium into cells restores the normal transmembrane potential and can be accomplished by injection of glucose and insulin or administration of a $\beta_{2}$-agonist (e.g., albuterol by nebulization) [8]. Sodium bicarbonate promotes the exchange of $\mathrm{K}^{+}$for hydrogen ions by causing extracellular alkalosis, although the relative effectiveness of this treatment option has been questioned [8].

If the body stores of potassium are excessive, as might be expected in a patient with renal failure, treat with sodium polystyrene sulfonate (e.g., Kayexalate) orally or by rectal tube and consider urgent hemodialysis.

\section{CASE CONTINUATION}

Based on the peaked $T$ waves and hyperkalemia, the patient was treated with intravenous insulin plus dextrose, sodium bicarbonate, and oral Kayexalate. Despite this regimen, the patient's $T$ waves did not normalize and she remained in what appeared to be an accelerated junctional rhythm. The serum digoxin level returned at $3.8 \mathrm{ng} / \mathrm{mL}$ (4.9 $\mathrm{nmol} / \mathrm{L})$. The last known ingestion of the drug was greater than 24 hours prior to arrival. At this point, the regional poison control center was contacted regarding the management of digoxin toxicity.

\section{Is this patient's cardiotoxicity a result of digoxin? Hyperkalemia? Both? What physiologic factors affect the relative toxicity of digoxin and hyperkalemia?}

The short answer is that this patient's cardiotoxicity reflects the effects of both digoxin and hyperkalemia. Exactly how these toxicities overlap, however, cannot be answered so easily and will require a more nuanced review of their effects. We will divide our further discussion into two parts based on the most salient problems posed by our case: digoxin toxicity and the role of the potassium level in patients with digoxin toxicity.

Digitalis and other cardioactive steroids occupy a unique role in the history of toxicology. The roots of the term "toxin," in fact, can be traced back to the Ouabaio tree and its botanical cousins. Ancient people from several cultures used ouabain-like extracts as a poisonous arrowhead coat-what the Greeks called toxikon. Thus, the modern field of toxicology owes its foundations, in part, to chemical warfare. Somewhat redeemingly, the ancient
Greeks, Romans and Egyptians also recognized the therapeutic value of cardioactive steroids. The Ebers Papyrus from Egypt circa 1500 BCE makes general reference to treatments with sea squill (or "sea onion") extract, a bufotoxin closely related to toad venom [12]. "Foxes glofa" was identified in the Welsh pharmaceutical book Meddygon Myddmai circa 1250 [12]. Modern medicine is most familiar with William Withering's report in 1785 of the use of Digitalis purpurea (foxglove) extracts for the treatment of "dropsy" (congestive heart failure) [12,13].

Digoxin toxicity is generally regarded as an extension of its pharmacologic effects. Bradycardia and atrioventricular block are thought to be mediated by the autonomic nervous system, via vagotonic effects, anti-adrenergic effects, or both [14,15]. There also appears to be a depressant effect on intrinsic sinus nodal automaticity not accounted for by pharmacologic blockade of the sympathetic and parasympathetic nervous systems [16].

Inhibition of $\mathrm{Na}^{+}-\mathrm{K}^{+}$-ATPase in cardiac cells by digoxin reduces the pumping of sodium out of the cell and potassium into the cell, leading to a buildup of sodium in the cytoplasm that reduces the driving force for calcium expulsion via the $\mathrm{Na}^{+}-$ $\mathrm{Ca}^{++}$exchanger. This increases the cytoplasmic calcium concentration, which in turn increases the storage of calcium in the sarcoplasmic reticulum, leading to enhanced actin-myosin coupling and myocyte contraction [12]. Increased levels of calcium within the conducting cells may also enhance excitability and result in delayed after-depolarizations [1].

Dysrhythmias associated with digoxin poisoning may also be caused or exacerbated by electrolyte derangements (see below).

\section{How do abnormal serum potassium levels affect the toxicity of digoxin?}

Experiments carried out in the 1970s and 1980s demonstrated that hypokalemia enhances digoxin toxicity by at least two mechanisms: increased cardiac glycoside binding to $\mathrm{Na}^{+}-\mathrm{K}^{+}-$ ATPase, and reduced renal clearance of digitalis glycosides due to increased proximal tubule reabsorption [17-19].

The converse relationship has also been proven, that is, moderate hyperkalemia tends to decrease the risk of digoxin toxicity [20]. Page and colleagues observed that "potassium administered concomitantly with ouabain exerted a protective effect against ouabain intoxication" strong enough to induce a two-fold increase in the fatal dose of ouabain [21]. In a separate study, only $27.4 \%$ of $\mathrm{Na}^{+}-\mathrm{K}^{+}$-ATPase activity was inhibited in hyperkalemic dogs (serum $\mathrm{K}^{+}$5.0-7.5 $\mathrm{mmol} / \mathrm{L}$ ) receiving digoxin, whereas $43.3 \%$ of $\mathrm{Na}^{+}-\mathrm{K}^{+}$-ATPase activity was inhibited in their normokalemic counterparts (hyperkalemia in the absence of digoxin did not alter inotropy or $\mathrm{Na}^{+}-\mathrm{K}^{+}$-ATPase activity relative to normokalemia in this study) [18]. Endogenous digitalis-like activity may be influenced by the potassium level as well. An in vitro study showed that the secretion of oubain from adrenal cortical cells was inhibited in a potassium-rich media [12].

While patients with acute digoxin toxicity usually present with hyperkalemia due to the extracellular buildup of potassium resulting from $\mathrm{Na}^{+}-\mathrm{K}^{+}$-ATPase inhibition, patients on digoxin 
chronically may present with hypokalemia due to adjunct therapy with potassium-wasting diuretics. Such patients may also suffer from hypomagnesemia, an effect not only of diuretics but of digoxin-mediated decreases in tubular magnesium reabsorption [22]. In turn, hypomagnesemia enhances digoxin toxicity by reducing the intrinsic activity of $\mathrm{Na}^{+}-\mathrm{K}^{+}$-ATPase [20]. In a case-control study of patients with therapeutic and toxic digoxin levels, toxic subjects had significantly lower serum magnesium levels and were significantly more likely to be taking diuretics. Perhaps surprisingly, given the reflexive association between digoxin toxicity and serum potassium derangement, the authors found that "magnesium deficiency was the most frequently identified significant electrolyte disturbance in relation to digoxin toxicity." The same study also demonstrated that digoxin toxicity occurs at relatively low concentrations in patients with low serum magnesium levels [23].

\section{How should this patient be treated?}

In our patient, the peaked $\mathrm{T}$ waves and absence of $\mathrm{P}$ waves suggest serious hyperkalemia, but the rhythm disturbance is also consistent with digoxin toxicity. Accelerated junctional rhythm is a fairly common manifestation of digitalis toxicity in patients with underlying atrial fibrillation [24,25], and while we do not know this patient's indication for taking digoxin, it is likely that she has a history of atrial fibrillation. Since elimination of digoxin depends on renal excretion, her elevated creatinine suggests she had accumulated digoxin after repeated daily dosing, although it is also possible that she intentionally ingested a large amount acutely.

Given the lack of response to initial measures to counteract hyperkalemia, the elevated digoxin level, and the characteristic dysrhythmia, it is appropriate to recommend treatment with anti-digoxin antibodies (Digibind or Digifab). Because magnesium deficiency can exacerbate digoxin toxicity, we should also measure the serum magnesium level and consider magnesium supplementation if it is low. (While clearly indicated for hypomagnesemic patients with or without digoxin toxicity, optimal dosing of intravenous magnesium has yet to be established. Beware that excessive doses of magnesium can hyperpolarize the transmembrane potential, thus exacerbating depressant effects on both the SA node and cardiac conduction, leading ultimately to sinus arrest or asystole [26].)

\section{CASE CONTINUED}

The patient remained in an accelerated junctional rhythm despite therapy. Unfortunately, neither brand of digoxin immune Fab was immediately available in the hospital. Treatment with intravenous calcium (10 $m L$ of calcium gluconate 10\%) was recommended to help stabilize the patient's cardiac rhythm pending arrival of digoxin immune Fab therapy.

\section{I was taught that administering calcium to digitalized patients was contraindicated, yet calcium was recommended in this case. What approach does the evidence support?}

Many, if not most, of us have been taught that digoxin toxicity is an absolute contraindication to treatment with intravenous calcium [27]. A toxicologic "taboo" has even built up around the subject: that calcium plus digoxin can induce a "stone heart." However, the strength of the evidence on which this taboo rests has eroded with progressive scrutiny. In this final section, we will take another look at this increasingly dubious dogma.

By most accounts the "stone heart" concept originated with a 1936 JAMA article by Bower and Mengle suggestively entitled, "The Additive Effect of Calcium and Digitalis" [28]. Although Bower's paper continues to be cited frequently in support of the "calcium-as-poison" cliché, the evidence it described is neither clear nor convincing. Bower opened his paper with two case reports of fatality following the administration of intravenous calcium to patients on a prior regimen of digitalis glycosides. These fatalities prompted Bower to conduct a crossover study in 10 dogs who were dosed with calcium and digalen (an intravenous digitalis preparation) in alternating sequences. Bower found that when administered after therapeutic doses of digalen, only $30-40 \%$ of the normally lethal amount of calcium was required to induce "an abrupt and dramatic cessation of heart action." While this study might seem quite reasonable in abstract form, its frailties are readily apparent on closer reading.

The first of Bower's two patients had developed a post-surgical condition vaguely described as "toxic irritation of the accelerator mechanism through sympathetic involvement." Her cause of death, which was preceded by generalized convulsions, was equally cryptic. Bower noted only that the "autopsy revealed nothing definite as to the cause of death." Furthermore, Bower neglected to provide the patient's serum potassium or calcium concentrations, and he did not describe the rate of calcium infusion. Bower did note that the second patient had been diagnosed with hyperparathyroidism, yet he also made no mention of the patient's serum calcium level.

What if both of Bower's patients were hypokalemic? Then neither case would be relevant to our central question of using calcium to treat hyperkalemic cardiotoxicity in digitalized patients. As Ahmed and colleagues affirmed, "the relevance of [Bower's] observations to hyperkalemic patients is hardly established" [29]. Ironically, Bower explicitly acknowledged that the toxicity he attributed to calcium could be entirely accounted for by hypokalemia, noting that "[prior studies show] that perfused hearts may be kept beating for long periods of time in oxygenated Ringer's solution but that if the potassium ions are removed the heart stops in systole."

Bower cited a 1932 study by Lieberman [30] in support of his own findings, despite the fact that Lieberman's findings clearly confound Bower's case. To quote from Lieberman's paper:

When injected slowly enough, perfectly enormous quantities of calcium gluconate can be disposed of by the animal without affecting it very much one way or the other. On the other hand, very rapid injection is quickly fatal in small doses.... It seems to me that here is an extremely practical point that cannot be overstressed. With rates of injection kept down to reasonable limits some of the unfortunate fatal accidents might not have taken place. 
In short, Lieberman proved that for intravenous calcium it is the rate, not the dose, which makes the poison. In a Lancet article published the year after Bower's paper, Rogen made exactly this point, using Lieberman's study to refute one of Bower's five references:

In man an intravenous injection of $4 \mathrm{cc}$ of a $10 \%$ solution of calcium chloride has produced dizziness, collapse, respiratory embarrassment, and generalized muscle spasm; recovery ensued in five minutes. The rate of injection was not reported but in view of later findings it is almost certain that the solution was given too rapidly. [31].

Previous reports and animal studies had suggested a potential additive or synergistic effect of co-administered digitalis and calcium. In 1927, Gold and Edwards reported a decrease in the average lethal dose of ouabain in dogs that had previously received intravenous calcium chloride in doses ranging from 10 $\mathrm{mg} / \mathrm{kg}$ to $113 \mathrm{mg} / \mathrm{kg}$. (The rate of calcium infusion was not reported but was apparently enough to induce "disturbance of the P-wave, QRS group, and T-wave, and with the larger dose ventricular tachycardia" even prior to any ouabain administration) [32]. However, the experiments were performed in only 5 dogs and serum potassium levels were not reported. Later studies provided evidence against the hypothesis of additive or synergistic effect between calcium and digitalis glycosides. In 1939, Smith and colleagues [33] administered infusions of calcium chloride $20 \%$ at rates of $0.35-3.5 \mathrm{~mL} / \mathrm{kg} / \mathrm{min}$ to $12 \mathrm{dogs}$ poisoned with half of a lethal dose of digitalis tincture. Three of the dogs died in ventricular fibrillation after 0.7-10.5 min, with serum calcium levels of $38.4-46.6 \mathrm{mg} / \mathrm{dL}(9.6-11.7 \mathrm{mmol} / \mathrm{L})$. However, control dogs given calcium alone without prior digitalis also had enhanced automaticity, including extrasystoles and ventricular fibrillation, at serum calcium levels of 30-65 mg/dL. A comparison of the 12 digitalis-plus-calcium animals with 10 control (calcium-only) animals revealed a slight but not statistically significant reduction in the concentrations of calcium required to cause dysrhythmias and the average serum calcium levels at the time of death. The authors concluded that there was no evidence that digitalis potentiates calcium by rendering the heart more susceptible to ventricular fibrillation. They argued against a synergistic effect for cardiac toxicity of calcium plus digitalis, suggesting at most a "partially additive effect." They wrote, "Our experiments suggest that the danger of injecting calcium into the digitalized patient is simply that of injecting calcium into any patient with cardiac disease ... certainly this danger cannot be great in practice, considering the widespread use of calcium intravenously."

In 1960, Lown reported that dogs given up to $90 \%$ of a toxic dose of ouabain had no dysrhythmias after administration of large doses of calcium (2.7 g over $30 \mathrm{~min}$, with a peak measured serum calcium level of $46.2 \mathrm{mg} / \mathrm{dL}$ [11.6 mmol/L]). Lown could provoke arrhythmias with calcium only when more than $95 \%$ of the toxic ouabain dose was given [34]. In reference to Bower's "widely quoted report" and other cited concerns about calcium- digitalis toxicity, Lown also pointed out that no deaths had been reported from the widespread use of calcium to determine the circulation time, "which has been frequently employed in critically digitalized patients" [34].

In 1970, Nola [35] reported a study involving 21 dogs given calcium chloride followed by acetylstrophanthidin (an injectable cardiac glycoside) until ventricular tachycardia occurred. Calcium was infused at a rate of $1-2 \mathrm{mEq} / \mathrm{min}(20-40 \mathrm{mg} / \mathrm{min})$ for 30 minutes. Dogs served as their own controls and received saline instead of calcium on control runs. An increased risk of cardiac glycoside-induced myocardial excitability (ventricular tachycardia) was seen only when the serum calcium level was greater than $15 \mathrm{mEq} / \mathrm{L}$ (7.5 mmol/L), which was $75 \%$ of the lethal serum calcium level in their animals. Moreover, in animals made only "moderately" hypercalcemic (average serum calcium $12.48 \mathrm{mEq} / \mathrm{L}$ [6.24 $\mathrm{mmol} / \mathrm{L}])$ the dose of acetylstrophanthidin required to produce ventricular tachycardia was not significantly different from control animals with normal calcium levels.

In 1999, Ghaemmaghami and Harchelroad described a study [36] that showed no increase in the rate of dysrhythmias or mortality in guinea pigs treated with intravenous calcium for digoxininduced hyperkalemia. And in 2004, Hack et al. reported no hastening of the time to asystole in pigs given a lethal dose of digoxin followed by calcium chloride $10 \mathrm{mg} / \mathrm{kg}$ (versus saline) at the onset of arrhythmias consistent with hyperkalemia [37].

Case reports of intravenous calcium administration for hyperkalemia in patients who were later found to have digitalis intoxication indicate no apparent harm from the use of calcium [38,39]. Kuhn reported that she performed a 30-year Medline review and was unable to find any report of adverse effects after the administration of calcium to hyperkalemic patients with possible digoxin poisoning [40].

It has been suggested that given the theoretical risks of calcium and the contradictory literature, perhaps the safest strategy should be to withhold this treatment [41] or to give it more slowly, say over 20-30 min [8]. Before addressing this compromise, it is worth reflecting on what we are trying to treat. Our patient presented with peaked $\mathrm{T}$ waves, absent $\mathrm{P}$ waves, and a junctional rhythm. She did not have ectopic ventricular beats or bidirectional ventricular tachycardia. From a mechanistic point of view, she showed evidence of depressed sinus node activity and $\mathrm{AV}$ nodal conduction, which could be due to the depressant effects of hyperkalemia on pacemaker function and cardiac impulse conduction, to the "vagotonic" effects of digoxin, or to both. She did not exhibit increased automaticity or excitability associated with excessive intracellular accumulation of calcium. In the absence of digitalis antibodies, it was prudent and appropriate to treat this patient with a drug that can restore pacemaker activity and improve conduction while enhancing the transmembrane potential and thus reducing membrane excitability: calcium. On the other hand, if she had presented with hyperkalemia and an elevated digoxin level, but without electrocardiographic evidence of critical hyperkalemia, it would be more appropriate to start with potassium-reducing measures such as glucose plus insulin and sodium polystyrene sulfonate (Kayexalate). 
In summary, treat digoxin toxicity with digoxin-specific antibodies when available. For patients with life-threatening hyperkalemia manifest by loss of $\mathrm{P}$ waves, and especially if there is QRS widening, we recommend treatment with intravenous calcium. On the other hand, if a patient with digitalis toxicity has multiple ectopic ventricular beats or runs of ventricular tachycardia, look for associated electrolyte problems such as hypokalemia and hypomagnesemia and correct these disorders while giving the digoxin immune Fab.

\section{What about the suggestion to give the calcium more slowly (over 20-30 minutes) if digoxin toxicity is suspected?}

As noted earlier, very large amounts of calcium can be tolerated if given slowly [30]. However, the point of giving calcium for hyperkalemia is the urgent reversal of critical cardiotoxic manifestations, which would imply rapid, not gradual, infusion. Since no good data exist to address this question, the decision regarding how fast to push the calcium will ultimately depend on the severity of the clinical situation. An ECG with agonal, wide-complex bradycardia, for instance, clearly calls for a more drastic approach compared to a tracing with only peaked $\mathrm{T}$ waves in an otherwise stable patient in sinus rhythm. Future research may provide more precise answers as to the ideal rate of infusion for calcium under various clinical conditions.

\section{CONCLUSION}

The prolific author and physician Richard Gordon once cracked that "the history of medicine is largely the substitution of ignorance by fallacies" [42]. The conflation of fallacy and ignorance in the case of digitalis plus calcium may be the acceptance of Bower's shaky case reports at the expense of Lieberman's stronger-and arguably more useful-finding that the most important determinant of calcium toxicity is the dose and rate of infusion, whether or not digitalis glycosides are present. While some of the timidity surrounding digitalis treatment is grounded in rational evidence, much of it still seems to reflect subjective interpretation insulated from empirical reality. As Blaustein notes, the ancient toxic taboos associated with cardiac glycosides "scared off many physicians and nearly relegated digitalis to oblivion before it was perspicaciously resurrected by [William] Withering" [12]. Like the "pseudoaxioms" [43] that epinephrine should never be injected into toes or that opioids will mask the abdominal exam findings in a surgical patient, the old digitalis-calcium "stone heart" taboo withers under a careful review of the evidence.

\section{REFERENCES}

1. Hondehem LM and Roden DM. Agents used in cardiac arrhythmias. In: Katzung BG, editor. Basic \& Clinical Pharmacology. 6th ed. Stamford, CT: Appleton \& Lange; 1995.

2. Parham WA Mehdirad AA, Biermann KM, Fredman CS. Hyperkalemia revisited. Tex Heart Inst J 2006;33:40-47.
3. Acker CG, Johnson JP, Palevsky PM, Greenberg A. Hyperkalemia in hospitalized patients: causes, adequacy of treatment, and results of an attempt to improve physician compliance with published therapy guidelines. Arch Intern Med 1998; 158:917-924.

4. Wrenn KD, Slovis CM, Slovis BS. The ability of physicians to predict hyperkalemia from the ECG. Ann Emerg Med 1991; 20:1229-1232.

5. Olson KR. Selected drugs and toxins and other causes of altered serum potassium. In: Olson KR et al., editors. Poisoning \& Drug Overdose. 5th ed. New York: McGraw-Hill, 2007. Table I27 , page 37.

6. Hollander-Rodriguez JC, Calvert JF. Hyperkalemia. Am Fam Physician 2006;73:283-290.

7. Perazella MA, Mahnensmith RL. Hyperkalemia in the elderly: drugs exacerbate impaired potassium homeostasis. J Gen Intern Med 1997;12(10):646-656.

8. Evans KJ, Greenberg A. Hyperkalemia: a review. J Intens Care Med 2005;20(5):272-290.

9. Hille B. Ionic Channels in Excitable Membranes. 2nd ed. Sunderland, MA: Sinauer, 1992, pp 460-461.

10. Guyton AC, Hall JE. Textbook of Medical Physiology. 9th ed. Philadelphia: WB Saunders, 1996, pp 375-380.

11. Beeler GW Jr ,Reuter H. Membrane calcium current in ventricular myocardial fibers. J Physiol 1970;207:191-209.

12. Blaustein MP. Physiological effects of endogenous ouabain: control of intracellular $\mathrm{Ca}^{2+}$ stores and cell responsiveness. Am J Physiol: Cell Physiol 1993;264(33): C1367-C1387.

13. Eichhorn EJ, Gheorghiade M. Digoxin-new perspective on an old drug. New Eng J Med 2002;347(18):1394-1395.

14. Alboni P, Shantha N, Filippi L, Pirani R, Preziosi S, Tomasi AM, Masoni A. Clinical effects of digoxin on sinus node and atrioventricular node function after pharmacologic autonomic blockade. Am Heart J 1984 Nov;108(5):1255-1261.

15. Reiffel JA, Bigger JT, Cramer M. Effects of digoxin on sinus nodal function before and after vagal blockade in patients with sinus nodal dysfunction: a clue to the mechanisms of the action of digitalis on the sinus node. Am J Cardiol 1979; 43:983-989.

16. Gomes JA, Kang PS, El-Sherif N. Effects of digitalis on the human sick sinus node after pharmacologic autonomic blockade. Am J Cardiol 1981;48:783-788.

17. Prindle, KH Jr, Skelton CA, Epstein SE, Marcus FI. Influence of extracellular potassium concentration on myocardial uptake and inotropic effect of tritiated digoxin. Circ Res 1971;28(3):337-345.

18. Goldman RH, Coltart DJ, Schweizer E, Snidow G, Harrison DC: The inotropic effects of digoxin in hyperkalemia: relation to $(\mathrm{Na}+-\mathrm{K}+)$-ATPase inhibition in the intact animal. Circulation 1973;48:830-838.

19. Meldgaard, LE, Steiness E, Waldorff S. Time course of ouabain uptake in isolated myocardial cells: dependence on extracellular potassium and calcium concentration. $\mathrm{Br} \mathrm{J}$ Pharmacol 1981;73(2):341-345. 
20. Macdonald JE, Struthers AD. What is the optimal serum potassium level in cardiovascular patients? J Am Coll Cardiol 2004;43(2):155-161.

21. Page E, Real JE. Interrelationships between cardiac effects of ouabain, hypocalcemia, and hyperkalemia. Circ Res 1955; 3:501-505.

22. Crippa G, Sverzellati E, Gieorgi-Pierfraceschi M, Carrara GC. Magnesium and cardiovascular drugs: interactions and therapeutic role. Ann Ital Med Int 1999;14(1):40-45.

23. Young IS, Goh EM, McKillop UH, Stanford CF, Nicholls DP, Trimble ER. Magnesium status and digoxin toxicity. Br J Clin Pharmacol 1991;32(6):717-721.

24. Marchlinski FE, Hook BG, Callans DJ. Which cardiac disturbances should be treated with digoxin immune Fab (ovine) antibody? Am J Emerg Med 1991 Mar;9(2 Suppl 1):24-28.

25. Ma G, Brady WJ, Pollack M, Chan TC. Electrocardiographic manifestations: digitalis toxicity. J Emerg Med 2001;20:145-152.

26. Kontani M, Hara A, Ohta S, Ikeda T. Hypermagnesemia induced by massive cathartic ingestion in an elderly woman without pre-existing renal dysfunction. Intern Med $\mathbf{2 0 0 5}$

May;44(5):448-452.

27. Lewin NA. Cardiac glycosides. In: Goldfrank LR, Flomenbaum NI, Lewin NA, Weisman RS, Howland MA, Hoffman RS, editors. Goldfrank's Toxicologic Emergencies. 6th ed. Stamford, CT: Appleton \& Lange, 1998, p. 798.

28. Bower JO ,Mengle HAK. The additive effect of calcium and digitalis. JAMA 1936;106(14):1151-1153.

29. Ahmed J, Weisberg LS. Hyperkalemia in dialysis patients. Sem Dialysis 2001;14(5):348-356.

30. Lieberman AL. Studies on calcium: VI. Some Interrelationships of the cardiac activities of calcium gluconate and scillaren-B. J Pharmacol Exp Ther 1933;47:183-192.

31. Rogen AS: Toxic manifestations of calcium therapy in heart failure. Lancet 1940;240(6216):452-453.
32. Gold H, Edwards DJ. The effects of ouabain on the heart in the presence of hypercalcemia. Am Heart J 1927;3:45-50.

33. Smith PK, Winkler AW, Hoff HE. Calcium and digitalis synergism: the toxicity of calcium salts injected into digitalized animals. Arch Intern Med 1939;64:322-329.

34. Lown B, Black H, Moore FD. Digitalis, electrolytes, and the surgical patient. Am J Cardiol 1960;6:309-337.

35. Nola GT, Pope S, Harrison DC. Assessment of the synergistic relationship between serum calcium and digitalis. Am Heart J 1970;79(4):499-507.

36. Ghaemmaghami CA, Harchelroad F. Dangers of intravenous calcium chloride in the treatment of digoxin-induced hyperkalemia-fact or fiction? (Abstract) Acad Emerg Med 1999;6(5):378.

37. Hack JB, Woody JH, Lewis DE, Brewer K, Meggs WJ. The effect of calcium chloride in treating hyperkalemia due to acute digoxin toxicity in a porcine model. Clin Toxicol

2004;40(4):337-342.

38. Fenton F, Smally AJ, Laut J. Hyperkalemia and digoxin toxicity in a patient with kidney failure. Ann Emerg Med 1996;28(4):440-441.

39. Van Deusen SK, Birkhahn RH, Gaeta TJ. Treatment of hyperkalemia in a patient with unrecognized digitalis toxicity. Clin Toxicol 2003;41:373-376.

40. Kuhn M. Digoxin, hyperkalemia and renal failure (Letter). Ann Emerg Med 1997;29(5):695-696.

41. Hoffman RS. What to do with case reports: is folly that succeeds folly nonetheless? (Editorial) J Toxicol Clin Toxicol 2003;41(4):377-379.

42. Gordon R. The Alarming History of Medicine: Amusing Anecdotes from Hippocrates to Heart Transplants. New York: St. Martin's Griffin Press, 1993.

43. Newman DH: Truth, and epinephrine, at our fingertips: unveiling the pseudoaxiom. Ann Emerg Med 2007;50:476-477. 